\title{
Article \\ Bifuzzy-Bilevel Programming Model: Solution and Application
}

\author{
Jiahao Chen $(\mathbb{D}$, Yujiao Jiang *(i) and Guang Wang (i) \\ School of Management, Shanghai University, Shanghai 200444, China; cjhsy111@shu.edu.cn (J.C.); \\ wangguang518@shu.edu.cn (G.W.) \\ * Correspondence: jiang_yujiao@shu.edu.cn; Tel.: +86-21-6613-4414-805
}

Citation: Chen, J.; Jiang, Y.; Wang, G. Bifuzzy-Bilevel Programming Model: Solution and Application. Symmetry 2021, 13, 1572. https://doi.org/ 10.3390/sym13091572

Academic Editor: José Carlos R. Alcantud

Received: 2 August 2021

Accepted: 17 August 2021

Published: 26 August 2021

Publisher's Note: MDPI stays neutral with regard to jurisdictional claims in published maps and institutional affiliations.

Copyright: (c) 2021 by the authors. Licensee MDPI, Basel, Switzerland. This article is an open access article distributed under the terms and conditions of the Creative Commons Attribution (CC BY) license (https:// creativecommons.org/licenses/by/ $4.0 /)$.

\begin{abstract}
Bi-level programming is widely used in processing various questions, but it cannot deal with the complex and fuzzy information contained in problems. In order to solve such problems better with intricate and vague information that can be efficiently handled by bifuzzy theory, a bifuzzybilevel programming model that sets the parameters to bifuzzy variables is proposed in this paper, which can process complex realistic data more accurately and improve the feasibility and validity of bi-level programming models. To ensure the solvability of the model, the equivalent form of the bifuzzy-bilevel programming model is obtained by utilizing the expected value operator. According to the linear and nonlinear characteristics of the model, the Karush-Kuhn-Tucker condition and particle swarm optimization algorithm are employed to handle the problem, respectively. Finally, by taking the distribution center location problem of the supplier as an example, the bifuzzybilevel programming model is applied in practice to balance highly intricate customer demands and corporate cost minimization, obtaining the feasible solution of functions at the upper and lower levels, and the bifuzzy information in the problem can also be processed well, which proves the effectiveness of the proposed methodology.
\end{abstract}

Keywords: bifuzzy variables; bi-level programming; Karush-Kuhn-Tucker condition; supply chain

\section{Introduction}

Bi-level programming was first proposed in the study of market competition in unbalanced economies. In 1973, bi-level programming first appeared to model a decentralized decision system of a leader and a follower with opposing interests in the paper of Bracken and McGill [1]. Bi-level programming studies the non-cooperative interaction between two decision makers with objective functions and coordinates decision contradictions. It is often used by scholars to describe an industry situation in which several groups are connected in a hierarchy. Each group can be an individual or an agent with separate and possibly conflicting goals [2].

In the past few decades, the applications of bi-level programming have greatly expanded, such as the production cost problem [3], traffic signal optimization [4], supply chain [5] and competitive electricity market applications [6]. It is difficult to solve bi-level programming problems because bi-level programming problems are NP-hard problems. To solve bi-level programming problems, many methods have been proposed such as tge imperialist competitive algorithm [7], genetic algorithm and particle swarm optimization algorithm [8]. However, the Karush-Kuhn-Tucker (KKT) condition is still the most commonly used method to solve linear bi-level programming issues.

In actual practice, the parameters or coefficients in bi-level programming are indeterminate. Some scholars have used fuzzy theory to deal with this indeterminate information. Emam [2] proposed a bi-level programming method involving a fuzzy approach to solve the problems of bi-level integer non-linear programming. Bi-level programming with fuzzy processing was often used to solve supply chain problems at first. With the improvement of the combination of the two methods, fuzzy bi-level programming was gradually ap- 
plied to water resources management [9], transportation [10], production and work force assignment [11] and plant location [12].

Considering that the information about fuzzy theory cannot be given accurately in reality, some researchers have focused on the extension of fuzzy theory, addressing two situations: one is incomplete information on the degree of membership, and the other is incomplete information on the objects of the universal set. To solve optimization problems with double uncertainty, the bifuzzy variable, which belongs to the latter case [13], was proposed by Liu [14] to represent the function from a possibility space to the set of fuzzy variables. In many practical issues, decision markers cannot tell us the precise requirements of problems, but they can give us their intervals. Using bifuzzy theory, people can obtain more benefits from personal experience and handle this complex uncertainty well in comparison to the fuzzy method [13]. Scholars have defined important concepts of bifuzzy variables [14] such as the expected value operator, chance measure and programming models. To solve the optimization problems with bifuzzy information, Zhou et al. [15] originally proposed the bifuzzy expected value model, bifuzzy chance-constrained programming and bifuzzy dependent-chance programming, which solved single-objective optimization problems with bifuzzy parameters.

In this paper, we study the combined application and solution of bifuzzy variables and bi-level programming with the aim of solving the problems with bifuzzy information in practice. The application scope of bifuzzy theory is extended, and the proposed method can solve bi-level programming problems with bifuzzy parameters. The expected value operator of bifuzzy variables is first applied to the upper and lower level models of the bi-level programming, and a bifuzzy-bilevel programming model is proposed. Then, the equivalent crisp model is obtained according to the expected value calculation formula proposed by Wang et al. [16], and the corresponding solution method is given according to the linear and nonlinear forms of the model. In order to verify the effectiveness of the proposed methodology, the model is applied to solve the distribution center location problem. The goals of solving a bi-level programming problem are to minimize the cost of distribution center construction and the customer service time. The bifuzzy parameters are involved in the formulation of bifuzzy-bilevel programming, and a satisfactory solution is obtained by employing the above process.

The rest of the paper is organized as follows. In Section 2, we review some concepts and theorems about LR fuzzy variables and bifuzzy variables. In Section 3, we introduce the general form of bifuzzy-bilevel programming and provide the solution method. The model is applied to an example of the distribution center location problem in Section 4 . Finally, conclusions are presented in Section 5.

\section{Preliminaries}

In this section, we introduce some useful definitions and theorems about LR fuzzy variables, bifuzzy variables and expected value operators of bifuzzy variables to help model the bi-level programming problem with bifuzzy parameters.

\subsection{LR Fuzzy Variables}

Definition 1 (Dubios and Prade [17]). Suppose that two shape functions are described as $L(\cdot)$ and $R(\cdot)$. If $L(\cdot)$ (or $R(\cdot))$ is a function that satisfies

(i) $L(0)=1$;

(ii) $L(x)<1, \forall x>0$;

(iii) $L(x)>0, \forall x<1$;

(iv) $L(1)=0$ or $[L(x)>0, \forall x$ and $L(+\infty)=0]$;

(v) $L(x)$ is nonincreasing on $[0,+\infty)$, 
and the fuzzy number $\tilde{\xi}$ has the following membership function:

$$
\mu_{\tilde{\xi}}(x)= \begin{cases}L\left(\frac{b-x}{\iota}\right), & \text { if } x \leq b \\ R\left(\frac{x-b}{v}\right), & \text { if } x>b,\end{cases}
$$

where $b$ is called the peak of $\tilde{\xi}$ with $\mu_{\tilde{\xi}}(b)=1$, and $\iota$ and $v$ are called the left and right spreads with $\iota>0$ and $v>0$, respectively; then, $\tilde{\xi}$ is said to be an LR fuzzy number. Symbolically, $\tilde{\zeta}$ is written as $(b, l, v)_{L R}$.

Example 1. If a triangular fuzzy number $\tilde{\xi}$ is described as $\mathcal{T}\left(a_{1}, a_{2}, a_{3}\right)$ with real numbers $a_{1}<$ $a_{2}<a_{3}$ and the shape functions satisfy $L(x)=R(x)=\max \{0,1-x\}$, then the membership function of $\tilde{\xi}$ is presented as

$$
\mu_{\tilde{\xi}}(x)= \begin{cases}\frac{x-a_{1}}{a_{2}-a_{1}}, & \text { if } a_{1}<x \leq a_{2} \\ \frac{a_{3}-x}{a_{3}-a_{2}}, & \text { if } a_{2}<x \leq a_{3} \\ 0, & \text { otherwise. }\end{cases}
$$

Definition 2 (Liu [18]). Suppose that $\Theta$ symbolizes a nonempty set, $\mathcal{P}(\Theta)$ stands for the power set of $\Theta$ and $\operatorname{Cr}$ represents the credibility measure, such that $\operatorname{Cr}\{\varnothing\}=0$ and $\operatorname{Cr}\{\Theta\}=1$. Then, the triplet $(\Theta, \mathcal{P}(\Theta), \mathrm{Cr})$ is called a credibility space.

Definition 3 (Liu and Liu [19]). Suppose that $\tilde{\xi}$ is a fuzzy number with the membership function $\mu$ and $r$ is a real number. The credibility measure can be written as

$$
\operatorname{Cr}\{\tilde{\zeta} \leq r\}=\left(\sup _{x \leq r} \mu(x)+1-\sup _{x \leq r} \mu(x)\right) / 2 .
$$

Definition 4 (Liu [18]). Suppose that the triplet $(\Theta, \mathcal{P}(\Theta), \mathrm{Cr})$ represents a credibility space with a fuzzy variable $\tilde{\xi}$ defined on it. Then, the credibility distribution of $\tilde{\xi}$ is defined as

$$
\mathrm{Y}_{\tilde{\xi}}(x)=\operatorname{Cr}\{\theta \in \Theta \mid \tilde{\xi}(\theta) \leq x\}, \quad \forall x \in \mathbb{R} .
$$

Example 2. On the basis of Definitions 3 and 4, the credibility distribution of the LR fuzzy number in Example 1 can be determined as

$$
\mathrm{Y}_{\tilde{\xi}}(x)= \begin{cases}0, & \text { if } x \leq a_{1} . \\ \frac{x-a_{1}}{2\left(a_{2}-a_{1}\right)}, & \text { if } a_{1}<x \leq a_{2} \\ 1-\frac{a_{3}-x}{2\left(a_{3}-a_{2}\right)}, & \text { if } a_{2}<x \leq a_{3} \\ 1, & \text { if } a_{3}<x\end{cases}
$$

Theorem 1 (Zhou et al. [20]). Suppose that the triplet $(\Theta, \mathcal{P}(\Theta), \mathrm{Cr})$ represents a credibility space with an LR fuzzy variable $\tilde{\xi}$ defined on it. If its credibility distribution is continuous and strictly increasing on its support, then $\tilde{\xi}$ is called a regular (LR) fuzzy variable.

Generally, if a regular (LR) fuzzy variable $\tilde{\xi}$ has the same shape functions (i.e., $L(\cdot)=R(\cdot)$ ) and same left and right spreads (i.e., $\iota=v$ ), we can call it symmetric [16]. The concept of the inverse credibility distribution plays a significant role for the regular fuzzy numbers. 
Definition 5 (Zhou et al. [20]). Let $\xi$ be a fuzzy number with a regular credibility distribution $\mathrm{Y}$. Then, the inverse function $\mathrm{Y}^{-1}$ is called the inverse credibility distribution of $\xi$.

Example 3. The inverse credibility distribution of a triangular fuzzy number $\tilde{\xi}$ described as $\mathcal{T}\left(a_{1}, a_{2}, a_{3}\right)$ is

$$
\mathrm{Y}^{-1}(\alpha)= \begin{cases}\left(2 a_{2}-2 a_{1}\right) \alpha+a_{1}, & \text { if } 0<\alpha \leq 0.5 \\ \left(2 a_{3}-2 a_{2}\right) \alpha+2 a_{2}-a_{3}, & \text { if } 0.5<\alpha<1\end{cases}
$$

Apparently, it should be noted that for the triangular fuzzy number defined in Example 3, when $a_{2}=0.5\left(a_{1}+a_{3}\right)$, it is symmetric [16].

\subsection{Bifuzzy Variables}

The bifuzzy variable is an extension of fuzzy numbers, and its definition and some elementary properties are discussed here as follows.

Definition 6 (Liu [18]). Suppose that the triplet $(\Theta, \mathcal{P}(\Theta), \mathrm{Cr})$ represents a credibility space. Then, a bifuzzy variable $\tilde{\tilde{\zeta}}$ defined on it can be regarded as a function from this space to the collection of fuzzy variables.

Example 4. If a variable $\tilde{\tilde{\zeta}}$ is given as $\mathcal{T}(\tilde{\xi}-\iota, \tilde{\zeta}, \tilde{\zeta}+v)$, where $\tilde{\zeta}$ represents a triangular fuzzy number $\mathcal{T}\left(a_{1}, a_{2}, a_{3}\right)$, then $\tilde{\tilde{\zeta}}$ is a bifuzzy variable. For example, if we take $a_{1}=1, a_{2}=2, a_{3}=3$ (i.e., $\tilde{\xi}=\mathcal{T}(1,2,3)$ ) and $\iota=v=1($ i.e., $\tilde{\tilde{\xi}}=\mathcal{T}(\tilde{\xi}-1, \tilde{\xi}, \tilde{\xi}+1)$ ), then $\tilde{\tilde{\xi}}$ is a bifuzzy variable with the domain $[0,4]$.

Definition 7 (Wang et al. [16]). A regular bifuzzy variable, $\tilde{\tilde{\xi}}$, is a regular LR fuzzy variable $\tilde{\xi}$ defined on the special set of regular LR fuzzy variables satisfying the following conditions:

(i) The shape functions are the same;

(ii) The left and right spreads are all denoted by two positive numbers $\iota$ and $v$;

(iii) For $\theta \in \Theta$, the peak of $\tilde{\tilde{\zeta}}(\theta)$ is determined by $\tilde{\xi}(\theta)$.

Obviously, the bifuzzy variable defined in Example 4 is regular. For simplicity, the general form of a regular bifuzzy variable is given below.

Definition 8 (Wang et al. [16]). Suppose that the triplet $(\Theta, \mathcal{P}(\Theta), \mathrm{Cr})$ represents a credibility space with a regular bifuzzy variable $\tilde{\tilde{\xi}}$ defined on it. If $\iota_{1}>0$ and $v_{1}>0$, the general form of $\tilde{\tilde{\xi}}$ can be written as $\tilde{\tilde{\xi}}=\left(\tilde{\xi}, \iota_{1}, v_{1}\right)_{L_{1} R_{1}}$, where $\tilde{\xi}=\left(b, \iota_{2}, v_{2}\right)_{L_{2} R_{2}}$ with real numbers $\iota_{2}>0$ and $v_{2}>0$. That is, the membership function of the primary fuzzy number $\tilde{\xi}$ is

$$
\mu_{\tilde{\xi}}(x)= \begin{cases}L_{2}\left(\frac{b-x}{\iota_{2}}\right), & \text { if } x \leq b \\ R_{2}\left(\frac{x-b}{v_{2}}\right), & \text { if } x>b\end{cases}
$$

and for any $\theta \in \Theta$, the membership function of the secondary fuzzy number $\tilde{\tilde{\zeta}}(\theta)$ is

$$
\mu_{\tilde{\tilde{\xi}}(\theta)}(x)= \begin{cases}L_{1}\left(\frac{\tilde{\xi}(\theta)-x}{\iota_{1}}\right), & \text { if } x \leq \tilde{\xi}(\theta) \\ R_{1}\left(\frac{x-\tilde{\xi}(\theta)}{v_{1}}\right), & \text { if } x>\tilde{\xi}(\theta) .\end{cases}
$$


In order to make the model computable, we introduce the expected value operator of regular bifuzzy variables, defined by Choquet integrals.

Theorem 2 (Wang et al. [16]). Suppose that the triplet $(\Theta, \mathcal{P}(\Theta), \mathrm{Cr})$ represents a credibility space with a regular bifuzzy variable $\tilde{\tilde{\xi}}$ defined on it, and the primary fuzzy number $\tilde{\xi}$ of $\tilde{\tilde{\xi}}$ has the inverse credibility distribution $\mathrm{Y}_{\tilde{\xi}}^{-1}$. Then, the expectation of $\tilde{\tilde{\xi}}$ is

$$
E[\tilde{\tilde{\zeta}}]=\int_{0}^{1} \int_{0}^{1} \mathrm{Y}_{\tilde{\zeta}}^{-1}(\alpha) \mathrm{d} \alpha \mathrm{d} \beta,
$$

where $\tilde{\zeta}$ is a secondary fuzzy number of $\tilde{\tilde{\xi}}$, expressed as $\left(\mathrm{Y}_{\tilde{\xi}}^{-1}(\beta), l, v\right)_{L R}$, with respect to $\beta$, and $\mathrm{Y}_{\tilde{\zeta}}^{-1}$ represents the inverse credibility distribution of $\tilde{\zeta}$.

Example 5. If $\tilde{\tilde{\xi}}$ is a triangular regular bifuzzy variable-i.e., $\tilde{\tilde{\xi}}=\mathcal{T}\left(\tilde{\xi}-\iota_{1}, \tilde{\xi}, \tilde{\xi}+v_{1}\right)$ with $\tilde{\xi}=\mathcal{T}\left(m-\iota_{2}, m, m+v_{2}\right)$-then the expectation of $\tilde{\tilde{\xi}}, E[\tilde{\tilde{\xi}}]$ can be deduced as

$$
\begin{aligned}
E[\tilde{\tilde{\xi}}]= & \int_{0}^{1} \int_{0}^{1} \mathrm{Y}_{\tilde{\zeta}}^{-1}(\alpha) \mathrm{d} \alpha \mathrm{d} \beta \\
= & \int_{0}^{0.5} \int_{0}^{0.5}\left(m+\iota_{2}(2 \beta-1)+\iota_{1}(2 \alpha-1)\right) \mathrm{d} \alpha \mathrm{d} \beta \\
& \quad+\int_{0}^{0.5} \int_{0.5}^{1}\left(m+\iota_{2}(2 \beta-1)-v_{1}(1-2 \alpha)\right) \mathrm{d} \alpha \mathrm{d} \beta \\
& \quad+\int_{0.5}^{1} \int_{0}^{0.5}\left(m-v_{2}(1-2 \beta)+\iota_{1}(2 \alpha-1)\right) \mathrm{d} \alpha \mathrm{d} \beta \\
& \quad+\int_{0.5}^{1} \int_{0.5}^{1}\left(m-v_{2}(1-2 \beta)-v_{1}(1-2 \alpha)\right) \mathrm{d} \alpha \mathrm{d} \beta \\
= & \frac{2 m-\iota_{1}-\iota_{2}}{8}+\frac{2 m+v_{1}-\iota_{2}}{8}+\frac{2 m-\iota_{1}+v_{2}}{8}+\frac{2 m+v_{1}+v_{2}}{8} \\
= & \frac{4 m-\iota_{1}+v_{1}-\iota_{2}+v_{2}}{4} .
\end{aligned}
$$

Definition 9 (Liu [21]). Suppose that $g\left(x_{1}, x_{2}, \cdots, x_{n}\right)$ is a real-valued function. If $g$ is strictly increasing with respect to $x_{1}, x_{2}, \cdots, x_{t}$ and strictly decreasing with respect to $x_{t+1}, x_{t+2}, \cdots, x_{n}$, then it is called strictly monotonous. That is, the following conditions should be satisfied simultaneously:

(i) For all $y_{i} \geq 0, i=1,2, \cdots, n$, we have

$$
g\left(x_{1}, x_{2}, \cdots, x_{n}\right) \leq g\left(x_{1}+y_{1}, \cdots, x_{t}+y_{t}, \cdots, x_{t+1}-y_{t+1}, \cdots, x_{n}-y_{n}\right) ;
$$

(ii) For all $y_{i}>0, i=1,2, \cdots, n$, we have

$$
g\left(x_{1}, x_{2}, \cdots, x_{n}\right)<g\left(x_{1}+y_{1}, \cdots, x_{t}+y_{t}, \cdots, x_{t+1}-y_{t+1}, \cdots, x_{n}-y_{n}\right) .
$$

Theorem 3 (Wang et al. [16]). Suppose that the triplet $(\Theta, \mathcal{P}(\Theta), C r)$ represents a credibility space with regular bifuzzy variables $\tilde{\tilde{\xi}}_{i}$ defined on it, and the primary fuzzy numbers $\tilde{\xi}_{i}$ of $\tilde{\tilde{\xi}}_{i}$ have the inverse credibility distributions $Y_{\tilde{\xi}_{i}}^{-1}, i=1,2, \cdots, n$. If $\tilde{\tilde{\xi}}_{1}, \tilde{\tilde{\xi}}_{2}, \ldots, \tilde{\xi}_{n}$ are independent and the function $g\left(x_{1}, x_{2}, \cdots, x_{n}\right)$ is continuous and strictly increasing with reference to $x_{1}, x_{2}, \cdots, x_{t}$ and strictly decreasing with reference to $x_{t+1}, x_{t+2}, \cdots, x_{n}$, then the expectation of $g\left(\tilde{\tilde{\xi}}_{1}, \tilde{\tilde{\xi}}_{2}, \cdots, \tilde{\tilde{\xi}}_{n}\right)$ is

$$
E\left[g\left(\tilde{\tilde{\xi}}_{1}, \tilde{\tilde{\zeta}}_{2}, \cdots, \tilde{\tilde{\zeta}}_{n}\right)\right]=\int_{0}^{1} \int_{0}^{1} g\left(\mathrm{Y}_{\tilde{\zeta}_{1}}^{-1}(\alpha), \cdots, \mathrm{Y}_{\tilde{\zeta}_{t}}^{-1}(\alpha), \mathrm{Y}_{\tilde{\zeta}_{t+1}}^{-1}(1-\alpha), \cdots, \mathrm{Y}_{\tilde{\zeta}_{n}}^{-1}(1-\alpha)\right) \mathrm{d} \alpha \mathrm{d} \beta,
$$


where $\tilde{\zeta}_{i}$ is a secondary fuzzy number of $\tilde{\tilde{\zeta}}_{i}$, expressed as

$$
\tilde{\zeta}_{i}= \begin{cases}\left(\mathrm{Y}_{\tilde{\xi}_{i}}^{-1}(\beta), \iota_{i}, v_{i}\right)_{L_{i} R_{i}}, & i=1,2, \cdots, t \\ \left(\mathrm{Y}_{\tilde{\xi}_{i}}^{-1}(1-\beta), \iota_{i}, v_{i}\right)_{L_{i} R_{i},} & i=t+1, t+2, \cdots, n,\end{cases}
$$

with respect to $\beta$.

Furthermore, the linearity of the expected value operator of independent bifuzzy variables has been mathematically proved.

Theorem 4 (Wang et al. [16]). Suppose that the triplet $(\Theta, \mathcal{P}(\Theta), C r)$ represents a credibility space with two independent regular bifuzzy variables $\tilde{\tilde{\xi}}_{1}$ and $\tilde{\tilde{\xi}}_{2}$ defined on it. If $E\left[\tilde{\tilde{\xi}}_{1}\right]$ and $E\left[\tilde{\tilde{\xi}}_{2}\right]$, the expected values of $\tilde{\tilde{\xi}}_{1}$ and $\tilde{\tilde{\xi}}_{2}$ are finite; then, for any two real numbers $m$ and $n$, we have

$$
E\left[m \tilde{\tilde{\xi}}_{1}+n \tilde{\tilde{\xi}}_{2}\right]=m E\left[\tilde{\tilde{\xi}}_{1}\right]+n E\left[\tilde{\tilde{\xi}}_{2}\right] .
$$

\section{Bifuzzy-Bilevel Programming}

In practice, uncertain information affects people's decision making. The influence of complex fuzzy information should also be considered when solving bi-level programming problems. In order to incorporate this information, we use bifuzzy parameters to describe the information, and a bifuzzy-bilevel programming model is constructed and studied in detail in this section.

\subsection{General Model}

In order to understand the general model of bifuzzy-bilevel programming, we introduce the definition of bi-level programming, which was presented by Bard [22].

Definition 10 (Bard [22]). For $x \in X \subset \mathbb{R}^{p}, y \in Y \subset \mathbb{R}^{q}$, a general bi-level programming problem is defined as

$$
\left\{\begin{array}{l}
\left.\min _{x \in X} F(\boldsymbol{x}, \boldsymbol{y}) \quad \text { (the upper level, leader }\right) \\
\text { subject to: } \\
\quad G(\boldsymbol{x}, \boldsymbol{y}) \leq 0 \\
\text { for each } \boldsymbol{x} \text { given by the leader, } \boldsymbol{y} \text { solves the follower's problem } \\
\quad \begin{array}{l}
\min _{\boldsymbol{y} \in Y} f(\boldsymbol{x}, \boldsymbol{y}) \quad \text { (the lower level, follower) } \\
\text { subject to: } \\
g(\boldsymbol{x}, \boldsymbol{y}) \leq 0
\end{array}
\end{array}\right.
$$

where $x, y$ are the decision variables of the upper level and the lower level, respectively; $F, f$ : $\mathbb{R}^{p} \times \mathbb{R}^{q} \rightarrow \mathbb{R}$ are the objective functions of the upper level and the lower level, respectively; and $G: \mathbb{R}^{p} \times \mathbb{R}^{q} \rightarrow \mathbb{R}^{m}, g: \mathbb{R}^{p} \times \mathbb{R}^{q} \rightarrow \mathbb{R}^{n}$ are the constraint conditions of the upper level and the lower level, respectively.

We now assume that, in a bi-level decision system, there is one leader and one follower. Let $x$ be the control vector of the leader and $y$ be that of the follower. Suppose that the cost objective function of the leader is $F(x, y, \tilde{\tilde{\xi}})$, where $\tilde{\tilde{\xi}}$ is a bifuzzy vector. Since the cost objective function contains bifuzzy parameters, it cannot be directly minimized. Instead, we minimize its expected value; i.e., $\min _{x} E[F(x, y, \tilde{\tilde{\xi}})]$. Similarly, we assume that the cost objective function of the follower is $f(x, y, \tilde{\tilde{\xi}})$, and we have $\min _{y} E[f(x, y, \tilde{\tilde{\xi}})]$. 
It should be noted that the bifuzzy constraint function of the leader is $G(x, y, \tilde{\tilde{\xi}}) \leq 0$. For each control vector $x$ chosen by the leader, the feasibility of control vector $y$ of the follower should be dependent on not only $x$ but also $y$. Similarly, we assume that the bifuzzy constraint function of the follower is $g(x, y, \tilde{\tilde{\xi}}) \leq 0$.

We assume that the leader first chooses their control vector $x$, and the follower determines their control vector $y$ after that. In the bifuzzy environment, to minimize the expected objectives of the leader and the follower, we have the following bifuzzy-bilevel programming problem:

$$
\left\{\begin{array}{l}
\min _{x} E[F(x, y, \tilde{\tilde{\xi}})] \\
\text { subject to: } \\
\quad E[G(x, y, \tilde{\tilde{\xi}})] \leq 0 \\
\quad M(x, y) \leq 0 \\
y \text { is the solution to the lower level problem } \\
\left\{\begin{array}{r}
\min _{y} E[f(x, y, \tilde{\tilde{\xi}})] \\
\text { subject to: } \\
E[g(x, y, \tilde{\tilde{\xi}})] \leq 0 \\
m(x, y) \leq 0
\end{array}\right.
\end{array}\right.
$$

in which $M(\boldsymbol{x}, \boldsymbol{y}) \leq 0, m(\boldsymbol{x}, \boldsymbol{y}) \leq 0$ are the crisp constraints of the leader and the follower, respectively.

\subsection{Equivalent Crisp Model}

From the mathematical viewpoint, there is no difference between deterministic mathematical programming and bifuzzy programming except for the fact that there are bifuzzy parameters in the latter. For the bifuzzy programming model (15), if the bifuzzy vector consists of regular LR bifuzzy numbers, a crisp equivalent form can be obtained by using Theorem 5 as follows.

Theorem 5. We assume that $\xi_{1}, \xi_{2}, \ldots, \xi_{n}$ are regular LR bifuzzy numbers; thus, the objective functions $F, f$ and the constraint functions $G, g$ are continuous n-ary real functions that strictly increase with respect to $\tilde{\tilde{\xi}}_{1}, \tilde{\tilde{\xi}}_{2}, \ldots, \tilde{\tilde{\xi}}_{k}$ and strictly decrease with respect to $\tilde{\tilde{\xi}}_{k+1}, \tilde{\tilde{\xi}}_{k+2}, \ldots, \tilde{\tilde{\xi}}_{n}$. Then, the bifuzzy-bilevel programming model (15) is equivalent to the crisp mathematical programming model:

$$
\left\{\begin{array}{l}
\min _{x} \int_{0}^{1} \int_{0}^{1} F\left(x, y, Y_{\tilde{\zeta}_{1}}^{-1}(\alpha), \ldots, Y_{\tilde{\zeta}_{k}}^{-1}(\alpha), Y_{\tilde{\zeta}_{k+1}}^{-1}(1-\alpha), \ldots, Y_{\tilde{\zeta}_{n}}^{-1}(1-\alpha)\right) \mathrm{d} \alpha \mathrm{d} \beta \\
\text { subject to: } \\
\quad \int_{0}^{1} \int_{0}^{1} G\left(x, y, Y_{\tilde{\zeta}_{1}}^{-1}(\alpha), \ldots, Y_{\tilde{\zeta}_{k}}^{-1}(\alpha), Y_{\tilde{\zeta}_{k+1}}^{-1}(1-\alpha), \ldots, Y_{\tilde{\zeta}_{n}}^{-1}(1-\alpha)\right) \mathrm{d} \alpha \mathrm{d} \beta \leq 0 \\
\quad M(x, y) \leq 0 \\
y \text { is the solution to the lower-level problem } \\
\left\{\begin{array}{c}
\min _{y} \int_{0}^{1} \int_{0}^{1} f\left(x, y, Y_{\tilde{\zeta}_{1}}^{-1}(\alpha), \ldots, Y_{\tilde{\zeta}_{k}}^{-1}(\alpha), Y_{\tilde{\zeta}_{k+1}}^{-1}(1-\alpha), \ldots, Y_{\tilde{\zeta}_{n}}^{-1}(1-\alpha)\right) \mathrm{d} \alpha \mathrm{d} \beta \\
\text { subject to: } \\
\quad \int_{0}^{1} \int_{0}^{1} g\left(x, y, Y_{\tilde{\zeta}}^{-1}(\alpha), \ldots, Y_{\tilde{\zeta}_{k}}^{-1}(\alpha), Y_{\tilde{\zeta}_{k+1}}^{-1}(1-\alpha), \ldots, Y_{\tilde{\zeta}_{n}}^{-1}(1-\alpha)\right) \mathrm{d} \alpha \mathrm{d} \beta \leq 0 \\
m(x, y) \leq 0
\end{array}\right.
\end{array}\right.
$$


where $\tilde{\zeta}_{i}$ are regular $L R$ fuzzy numbers, which can be expressed as

$$
\tilde{\zeta}_{i}= \begin{cases}\left(\mathrm{Y}_{\tilde{\xi}_{i}}^{-1}(\beta), \iota_{i}, v_{i}\right), & i=1,2, \cdots, t \\ \left(\mathrm{Y}_{\tilde{\xi}_{i}}^{-1}(1-\beta), \iota_{i}, v_{i}\right), & i=t+1, t+2, \cdots, n,\end{cases}
$$

with respect to $\beta$, as described in Theorem 3.

Proof of Theorem 5. It follows from Definition 5 that the inverse credibility distribution of $F\left(x, y, \tilde{\tilde{\zeta}}_{1}, \tilde{\tilde{\zeta}}_{2}, \cdots, \tilde{\tilde{\zeta}}_{n}\right)$ is $F\left(x, y, \mathrm{Y}_{\tilde{\zeta}_{1}}^{-1}(\alpha), \ldots, \mathrm{Y}_{\tilde{\zeta}_{k}}^{-1}(\alpha), \mathrm{Y}_{\tilde{\zeta}_{k+1}}^{-1}(1-\alpha), \ldots, \mathrm{Y}_{\tilde{\zeta}_{n}}^{-1}(1-\alpha)\right)$. Using Theorem 3 , we have $E\left[F\left(x, y, \tilde{\tilde{\zeta}}_{1}, \ldots, \tilde{\tilde{\zeta}}_{n}\right)\right]=\int_{0}^{1} \int_{0}^{1} F\left(x, y, \mathrm{Y}_{\tilde{\zeta}_{1}}^{-1}(\alpha), \ldots, \mathrm{Y}_{\tilde{\zeta}_{k}}^{-1}(\alpha), \mathrm{Y}_{\tilde{\zeta}_{k+1}}^{-1}\right.$ $\left.(1-\alpha), \ldots, \mathrm{Y}_{\tilde{\zeta}_{n}}^{-1}(1-\alpha)\right) \mathrm{d} \alpha \mathrm{d} \beta$. Similarly, the functions $f, G$ and $g$ can perform the same transformation.

In order to construct the bifuzzy-bilevel programming model (15), we simply need to find a numerical method for the deterministic mathematical programming model (16). As mentioned above, the bi-level programming problem is an NP-hard problem. Since this paper synthesizes bifuzzy parameters and bi-level programming, the solution of this problem can be more comprehensive than either of those techniques alone. If the objective functions and constraints of the deterministic mathematical programming model (16) are linear, we can use the classical KKT condition to solve the problem [23]. The KKT condition is used to transform the lower-level model, which cause bi-level programming to become single-level programming. The specific solving process of this method is illustrated in detail in Section 4.

If the objective functions and constraints of deterministic mathematical programming (16) are nonlinear, we integrate bifuzzy simulation and the particle swarm optimization algorithm to solve the problem $[15,24]$ :

Step 1. Initialize parameters such as the initial population of particles, the pbest solution and the fitness, the maximum and minimum velocity levels, the inertia weight, acceleration coefficients, the maximum iteration number and the current iteration number;

Step 2. Compute the fitness value via bifuzzy simulation and solve the lower level problem, then obtain and update the pbest solution for each particle;

Step 3. Update the gbest solution and introduce the gbest solution into the objective function of the upper level;

Step 4. If the current iteration number does not exceed the maximum iteration number, go tp Step 5; otherwise, stop and submit the current gbest solution as the optimum for the bi-level programming problem;

Step 5. Update the inertia weight, the velocity and the position of each particle. If the current velocity is less than the minimum velocity, set the current velocity equals to the minimum velocity; if the current velocity exceeds the maximum velocity, set the current velocity equal to the maximum velocity. Then, go to Step 2.

\section{Linear Case}

In this section, we apply the bifuzzy-bilevel programming model to the distribution center location problem of the supplier and verify its effectiveness. Proceeding from reality, the model aims to maximize customer satisfaction while minimizing the cost of the suppliers.

\subsection{Bifuzzy-Bilevel Programming Model for Distribution Center Location}

The distribution center location problem of the supplier is shown in Figure 1, which is described as a bifuzzy-bilevel programming problem. The upper level considers minimizing the construction and management cost of the distribution centers of the supplier. 
To meet the uncertain demands of customers in different regions, the supplier needs to establish distribution centers in corresponding regions, which will inevitably increase the capital investment of the supplier in warehousing equipment, transportation equipment and other aspects. Therefore, the supplier needs to minimize the cost of the construction process in the distribution center to ensure the full use of its funds $[25,26]$.

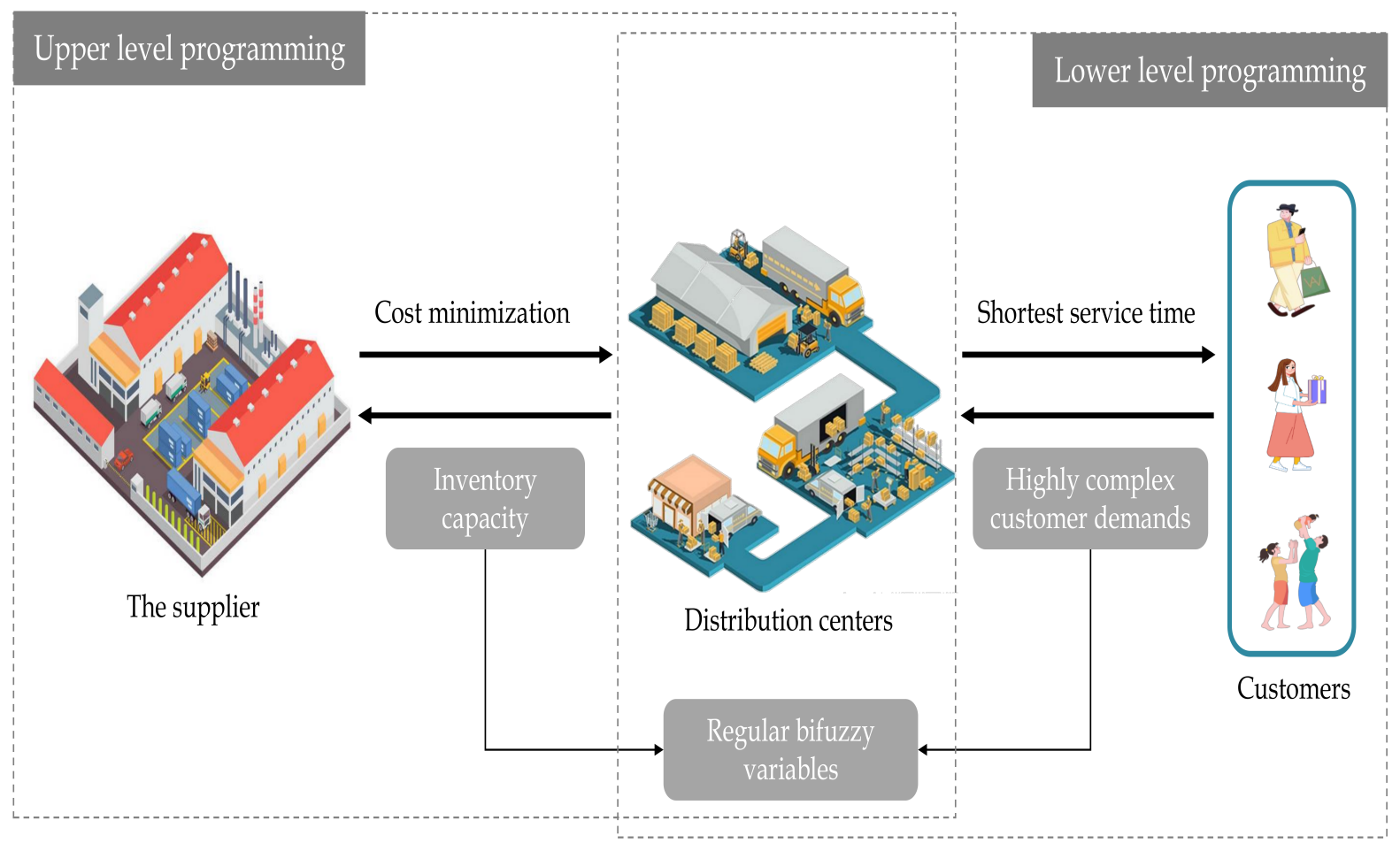

Figure 1. The distribution center location problem of the supplier.

The lower-level model aims to minimize the customer service time. Due to the overall competition environment, customers have higher requirements for timely delivery. The shorter the service time within the specified time limit, the greater the customer satisfaction will be.

The decision variables and parameters of this model are provided in Table 1. Because the customer demands are uncertain, bifuzzy variables are adopted for processing. Subsequently, the inventory capacity of distribution centers should consider the uncertainty of customer demands, for which bifuzzy variable processing is also adopted. Thus, among the model parameters, $\tilde{V}_{i}$ and $\tilde{W}_{j}$ are assumed to be bifuzzy variables.

The bifuzzy-bilevel programming model of this problem is expressed as follows:

$$
\left\{\begin{array}{l}
\min U=\sum_{i=1}^{n} C_{i} X_{i}+\theta \sum_{i=1}^{n} \sum_{j=1}^{m}\left(\tilde{V}_{i}-Y_{i j}\right) \\
\text { subject to: } \\
\quad \sum_{i=1}^{n} X_{i} \geq 1 \\
\sum_{j=1}^{m} Y_{i j} \leq X_{i} \tilde{V}_{i} \quad i=1,2, \ldots, n \\
X_{i} \in\{0,1\} \quad i=1,2, \ldots, n \\
\left\{\begin{array}{c}
\min L=\sum_{i=1}^{n} \sum_{j=1}^{m} Y_{i j} / \varphi_{i j} \\
\text { subject to: } \\
\sum_{i=1}^{n} Y_{i j} \leq \tilde{W}_{j}, \quad j=1,2, \ldots, m \\
Y_{i j} \geq 0, \quad i=1,2, \ldots, n ; j=1,2, \ldots, m .
\end{array}\right.
\end{array}\right.
$$


Table 1. Notations and explanations.

\begin{tabular}{|c|c|}
\hline Symbol & Explanations \\
\hline \multicolumn{2}{|c|}{ Decision variables } \\
\hline$X_{i}$ & $\begin{array}{l}\text { A binary integer variable, which represents whether distribution } \\
\text { center } i \text { is selected, } i=1,2, \ldots, n\end{array}$ \\
\hline$Y_{i j}$ & $\begin{array}{l}\text { The volume of product traffic from distribution center } i \text { to cus- } \\
\text { tomer } j, i=1,2, \ldots, n, j=1,2, \ldots, m\end{array}$ \\
\hline \multicolumn{2}{|r|}{ ( } \\
\hline$C_{i}$ & The operation and maintenance cost of distribution center $i$ \\
\hline$\varphi_{i j}$ & The efficiency of customer $j$ served by distribution center $i$ \\
\hline$\theta$ & $\begin{array}{l}\text { The punishment coefficient, which is the penalty for the insuffi- } \\
\text { cient inventory capacity utilization of distribution centers }\end{array}$ \\
\hline$\tilde{\tilde{V}}_{i}$ & $\begin{array}{l}\text { A regular bifuzzy variable, which represents the maximum stock } \\
\text { capacity of distribution center } i\end{array}$ \\
\hline$\tilde{\tilde{W}}_{j}$ & $\begin{array}{l}\text { A regular bifuzzy variable, which represents the total demand of } \\
\text { customer } j\end{array}$ \\
\hline
\end{tabular}

The objective function of the upper level includes the total operation and maintenance cost of the distribution center and the penalty cost for the underutilization of the inventory of the distribution center. The constraint contained in the upper level is that the supplier needs to build at least one distribution center and each distribution center has a maximum inventory limit. The objective function of the lower level is the total service time from distribution centers to customers. The constraint is that the total amount of products obtained by customers from the distribution center must not exceed the customers' demands.

\subsection{The Equivalent Crisp Programming Model}

Since the established model (18) is the cost objective function, we must minimize the expected value of the objective function to obtain the result according to model (15). This, we have the general model for this location problem:

$$
\left\{\begin{array}{l}
\min E\left[\sum_{i=1}^{n} C_{i} X_{i}+\theta \sum_{i=1}^{n} \sum_{j=1}^{m}\left(\tilde{V}_{i}-Y_{i j}\right)\right] \\
\text { subject to: } \\
\quad \sum_{i=1}^{n} X_{i} \geq 1 \\
E\left[\sum_{j=1}^{m} Y_{i j} \leq X_{i} \tilde{V}_{i}\right], \quad i=1,2, \ldots, n \\
X_{i} \in\{0,1\}, \quad i=1,2, \ldots, n \\
\left\{\begin{array}{c}
\min \sum_{i=1}^{n} \sum_{j=1}^{m} Y_{i j} / \varphi_{i j} \\
\text { subject to: } \\
E\left[\sum_{i=1}^{n} Y_{i j} \leq \tilde{W}_{j}\right], \quad j=1,2, \ldots, m \\
Y_{i j} \geq 0, \quad i=1,2, \ldots, n ; j=1,2, \ldots, m .
\end{array}\right.
\end{array}\right.
$$


Since the cost objective function and constraints of the bifuzzy-bilevel programming model are linear, the following model can be obtained according to Theorem 4 .

$$
\left\{\begin{array}{l}
\min \sum_{i=1}^{n} C_{i} X_{i}+\theta \sum_{i=1}^{n} \sum_{j=1}^{m}\left(E\left[\tilde{V}_{i}\right]-Y_{i j}\right) \\
\text { subject to: } \\
\quad \sum_{i=1}^{n} X_{i} \geq 1 \\
\sum_{j=1}^{m} Y_{i j} \leq X_{i} E\left[\tilde{V}_{i}\right], \quad i=1,2, \ldots, n \\
X_{i} \in\{0,1\}, \quad i=1,2, \ldots, n \\
\left\{\begin{array}{c}
\min \sum_{i=1}^{n} \sum_{j=1}^{m} Y_{i j} / \varphi_{i j} \\
\text { subject to: } \\
\sum_{i=1}^{n} Y_{i j} \leq E\left[\tilde{W}_{j}\right], \quad j=1,2, \ldots, m \\
Y_{i j} \geq 0, \quad i=1,2, \ldots, n ; j=1,2, \ldots, m .
\end{array}\right.
\end{array}\right.
$$

Then, we can convert model (20) to the equivalent crisp programming model according to Theorem 5,

$$
\left\{\begin{array}{l}
\min \sum_{i=1}^{n} C_{i} X_{i}+\theta \sum_{i=1}^{n} \sum_{j=1}^{m}\left(\int_{0}^{1} \int_{0}^{1} \mathrm{Y}_{\tilde{V}_{i}}^{-1}(\alpha) \mathrm{d} \alpha \mathrm{d} \beta-Y_{i j}\right) \\
\text { subject to: } \\
\quad \sum_{i=1}^{n} X_{i} \geq 1 \\
\sum_{j=1}^{m} Y_{i j} \leq X_{i} \int_{0}^{1} \int_{0}^{1} \mathrm{Y}_{\tilde{V}_{i}}^{-1}(\alpha) \mathrm{d} \alpha \mathrm{d} \beta, \quad i=1,2, \ldots, n \\
X_{i} \in\{0,1\}, \quad i=1,2, \ldots, n \\
\left\{\begin{array}{l}
\min \sum_{i=1}^{n} \sum_{j=1}^{m} Y_{i j} / \varphi_{i j} \\
\text { subject to: } \\
\sum_{i=1}^{n} Y_{i j} \leq \int_{0}^{1} \int_{0}^{1} \mathrm{Y}_{\tilde{\mathrm{W}}_{j}}^{-1}(\alpha) \mathrm{d} \alpha \mathrm{d} \beta, \quad j=1,2, \ldots, m \\
Y_{i j} \geq 0, \quad i=1,2, \ldots, n ; j=1,2, \ldots, m .
\end{array}\right.
\end{array}\right.
$$

The KKT condition is adopted to transform the model into a single-level mixed integer linear programming problem. This method is also the most widely accepted approach In general, the lower level is replaced by the KKT condition, and the bi-level model is replaced by a single-level model. Here, the KKT condition can be introduced to represent the linear programming relaxation of the lower level. $\lambda_{j}$ and $\mu_{i j}$ are, respectively, the Lagrange multipliers corresponding to constraint $\sum_{i=1}^{n} Y_{i j} \leq \int_{0}^{1} \int_{0}^{1} Y_{\tilde{W}_{j}}^{-1}(\alpha) \mathrm{d} \alpha \mathrm{d} \beta, Y_{i j} \geq 0$. The Lagrange function of the lower level programming is

$$
L\left(Y_{i j}, \lambda_{j}, \mu_{i j}\right)=\sum_{i=1}^{n} \sum_{j=1}^{m} Y_{i j} / \varphi_{i j}+\sum_{j=1}^{m} \lambda_{j}\left(Y_{i j}-\int_{0}^{1} \int_{0}^{1} Y_{\tilde{W}_{j}}^{-1}(\alpha) \mathrm{d} \alpha \mathrm{d} \beta\right)+\sum_{i=1}^{n} \sum_{j=1}^{m} \mu_{i j}\left(-Y_{i j}\right) .
$$

According to the standard form of KKT condition, the optimal solution of the lower level programming can be solved by the following constraints:

$$
\begin{gathered}
\sum_{i=1}^{n} \sum_{j=1}^{m} 1 / \varphi_{i j}+\sum_{j=1}^{m} \lambda_{j}-\sum_{i=1}^{n} \sum_{j=1}^{m} \mu_{i j}=0 \\
\left\{\begin{array}{l}
\mu_{i j} Y_{i j}=0 \\
\mu_{i j} \leq 0 \\
Y_{i j} \geq 0 \\
i=1,2, . ., n ; j=1,2, \ldots, m .
\end{array}\right.
\end{gathered}
$$




$$
\left\{\begin{array}{l}
\lambda_{j}\left(\sum_{i=1}^{n} Y_{i j}-\int_{0}^{1} \int_{0}^{1} \mathrm{Y}_{\tilde{W}_{j}}^{-1}(\alpha) \mathrm{d} \alpha \mathrm{d} \beta\right)=0 \\
\lambda_{j} \geq 0 \\
\sum_{i=1}^{n} Y_{i j}-\int_{0}^{1} \int_{0}^{1} \mathrm{Y}_{\tilde{W}_{j}}^{-1}(\alpha) \mathrm{d} \alpha \mathrm{d} \beta \leq 0 \\
j=1,2, \ldots, m .
\end{array}\right.
$$

Although the bifuzzy-bilevel programming model of the supply chain can be transformed by the KKT condition, the resulting programming contains nonlinear constraints (24) and (25). These constraints can be linearized by combining the properties of $0-1$ decision variables and the big $\mathrm{M}$ method by introducing binary variables $\left(b_{1}, b_{2}\right)$ and a large constant $M$ :

$$
\left\{\begin{array}{l}
-\left(1-b_{1}\right) M \leq \mu_{i j} \leq 0 \\
0 \leq Y_{i j} \leq b_{1} M \\
0 \leq \lambda_{j} \leq b_{2} M \\
-\left(1-b_{2}\right) M \leq \sum_{i=1}^{n} Y_{i j}-\int_{0}^{1} \int_{0}^{1} Y_{\tilde{W}_{j}}^{-1}(\alpha) \mathrm{d} \alpha \mathrm{d} \beta \leq 0 \\
i=1,2, . ., n ; j=1,2, \ldots, m .
\end{array}\right.
$$

Through the above steps, the bifuzzy-bilevel programming model is transformed into model (27):

$$
\begin{aligned}
& \left\{\begin{array}{l}
\min \sum_{i=1}^{n} C_{i} X_{i}+\theta \sum_{i=1}^{n} \sum_{j=1}^{m}\left(\int_{0}^{1} \int_{0}^{1} Y_{\tilde{V}_{i}}^{-1}(\alpha) \mathrm{d} \alpha \mathrm{d} \beta-Y_{i j}\right) \\
\text { subject to: }
\end{array}\right. \\
& \sum_{i=1}^{n} X_{i} \geq 1 \\
& \sum_{j=1}^{m} Y_{i j} \leq X_{i} \int_{0}^{1} \int_{0}^{1} \mathrm{Y}_{\tilde{V}_{i}}^{-1}(\alpha) \mathrm{d} \alpha \mathrm{d} \beta, \quad i=1,2, \ldots, n \\
& \sum_{i=1}^{n} Y_{i j} \leq \int_{0}^{1} \int_{0}^{1} Y_{\tilde{W}_{j}}^{-1}(\alpha) \mathrm{d} \alpha \mathrm{d} \beta, \quad j=1,2, \ldots, m \\
& \sum_{i=1}^{n} \sum_{j=1}^{m} 1 / \varphi_{i j}+\sum_{j=1}^{m} \lambda_{j}-\sum_{i=1}^{n} \sum_{j=1}^{m} \mu_{i j}=0 \\
& -\left(1-b_{2}\right) M \leq \sum_{i=1}^{n} Y_{i j}-\int_{0}^{1} \int_{0}^{1} Y_{\tilde{W}_{j}}^{-1}(\alpha) \mathrm{d} \alpha \mathrm{d} \beta \leq 0, \quad j=1,2, \ldots, m \\
& 0 \leq Y_{i j} \leq b_{1} M, \quad i=1,2, \ldots, n ; j=1,2, \ldots, m \\
& -\left(1-b_{1}\right) M \leq \mu_{i j} \leq 0, \quad i=1,2, \ldots, n ; j=1,2, \ldots, m \\
& 0 \leq \lambda_{j} \leq b_{2} M, \quad j=1,2, \ldots, m \\
& X_{i} \in\{0,1\}, \quad i=1,2, \ldots, n \text {. }
\end{aligned}
$$

Then, we input the data into model (27), use the Matlab yalmip programming solution to solve the model and obtain the minimum value of the cost objective function.

\subsection{Numerical Experiment}

The specific description of the example is as follows. An supplier needs to select the reasonable locations from 60 potential distribution centers to meet the uncertain demands of 120 customers. The related data are characterized in Tables 2 and 3. 
Table 2. The values of model parameters.

\begin{tabular}{llrr}
\hline Parameters & Scale & Value & Expected Value \\
\hline \multirow{2}{*}{$C_{i}$} & Small & 10 & - \\
\cline { 2 - 4 } & Medium & 20 & - \\
\cline { 2 - 4 } & Large & 30 & 300 \\
\hline \multirow{2}{*}{$\tilde{V}_{i}$} & Small & $\tilde{V}_{i}=\left(\tilde{V}_{i}, 60,60\right) ; \tilde{V}_{i}=(300,60,60)$ & 400 \\
\cline { 2 - 4 } & Medium & $\tilde{V}_{i}=\left(\tilde{V}_{i}, 60,60\right) ; \tilde{V}_{i}=(400,60,60)$ & 500 \\
\cline { 2 - 4 } & Large & $\tilde{V}_{i}=\left(\tilde{V}_{i}, 60,60\right) ; \tilde{V}_{i}=(500,60,60)$ & 130 \\
\hline$\tilde{W}_{j}$ & - & $\tilde{W}_{i}=\left(\tilde{W}_{i}, 30,30\right) ; \tilde{W}_{i}=(130,30,30)$ & - \\
\hline$\theta$ & - & & 2.5 \\
\hline
\end{tabular}

Table 3. The value of service efficiency.

\begin{tabular}{lrrrrrrrrr}
\hline $\begin{array}{l}\text { Service } \\
\text { Efficiency }\left(\varphi_{i j}\right)\end{array}$ & $\mathbf{1}$ & $\mathbf{2}$ & $\mathbf{3}$ & $\mathbf{4}$ & $\mathbf{5}$ & $\ldots$ & $\mathbf{5 8}$ & $\mathbf{5 9}$ & $\mathbf{6 0}$ \\
\hline 1 & & & & & & & & & \\
2 & $1 / 5$ & $1 / 2$ & $1 / 9$ & $1 / 9$ & $1 / 10$ & $\ldots$ & $1 / 5$ & $1 / 4$ & $1 / 2$ \\
3 & $1 / 5$ & $1 / 8$ & 1 & $1 / 7$ & $1 / 9$ & $\ldots$ & 1 & $1 / 5$ & $1 / 5$ \\
4 & 1 & $1 / 7$ & $1 / 3$ & $1 / 7$ & $1 / 5$ & $\ldots$ & $1 / 10$ & $1 / 4$ & $1 / 2$ \\
5 & $1 / 3$ & $1 / 7$ & $1 / 5$ & $1 / 9$ & $1 / 5$ & $\ldots$ & $1 / 5$ & $1 / 5$ & $1 / 7$ \\
$\vdots$ & $1 / 7$ & $1 / 10$ & $1 / 10$ & $1 / 2$ & $1 / 8$ & $\ldots$ & $1 / 5$ & $1 / 3$ & $1 / 3$ \\
118 & $\vdots$ & $\vdots$ & $\vdots$ & $\vdots$ & $\vdots$ & & $\vdots$ & $\vdots$ & $\vdots$ \\
119 & $1 / 2$ & $1 / 4$ & 1 & $1 / 2$ & $1 / 9$ & $\ldots$ & $1 / 5$ & $1 / 6$ & $1 / 3$ \\
120 & $1 / 4$ & $1 / 10$ & $1 / 10$ & $1 / 7$ & $1 / 5$ & $\ldots$ & $1 / 7$ & $1 / 6$ & $1 / 9$ \\
\hline
\end{tabular}

The specific values of the parameters used in the model are shown in Table 2. Table 3 shows the service efficiency from distribution centers to customers. Here, the inventory capacity of a distribution center and each customer's demands are regular bifuzzy numbers. To be closer to the actual situation, the size and cost of the distribution center are divided into three sizes, which are considered in 60 distribution centers. For example, the top 20 distribution centers are small, and the corresponding cost of construction is small, as well as that of satisfying nearby customer demands. $\tilde{\tilde{W}}_{j}$ equals $\left(\tilde{W}_{j}, 30,30\right)$ and the primary fuzzy number $\tilde{W}_{j}$ is $(130,30,30)$, which means the demands for each customer fluctuates between 100 and 160. The construction of the distribution center should match the corresponding capacity. For example, the medium scale of $\tilde{V}_{i}$ equals $\left(\tilde{V}_{j}, 60,60\right)$ and the primary fuzzy number $\tilde{V}_{j}$ is $(400,60,60)$, which shows that the capacity of the distribution center is between 340 and 460 .

A satisfactory solution for the target function is obtained after running Matlab for $28.62 \mathrm{~s}$. According to the results, 43 distribution centers are established, and locations 23, $41,42,44-46$ and 48-58 are not selected. The established distribution centers can meet the product needs of each customer without wasting the inventory of the distribution center. The optimal value of the converted objective function is 21,700.

For the distribution center location problem of the supplier, this section establishes and evaluates a bi-level programming model with bifuzzy parameters. Since the bifuzzy parameters are linear, we can use the expected value operator for the crisp processing of the bifuzzy-bilevel programming model and transform it into a general bi-level programming model which can be solved by the classic KKT condition. The satisfactory solution of the target function verifies that the proposed methodology can easily and effectively deal with real problems with bifuzzy characteristics. 


\section{Conclusions}

In this paper, we propose a bifuzzy-bilevel programming model and give the corresponding solution methods. To validate and illustrate the effectiveness of the proposed model, we apply it to solve the distribution center location problem. The main contributions of this research can be described as follows. Firstly, we combine bifuzzy theory with bi-level programming to propose the general bifuzzy-bilevel programming model and obtain the equivalent crisp model, further expanding the rationality of the application of bi-level programming. Secondly, on the foundation of the linear and nonlinear characteristics of the model, we give two different solving methods. Finally, we verify the effectiveness of the bifuzzy-bilevel programming model through an example.

The bifuzzy-bilevel programming model can be applied to other scenarios: for example, sales channels distribution problems, road planning problems and logistic network design problems, etc. In the future, it is worth investigating the utilization of bifuzzy variables to describe the complex fuzzy information in other programming problems and explore different ways to deal with the models with bifuzzy parameters. This paper only shows the linear solving process in detail, and the nonlinear solving process can also be further explored.

Author Contributions: Conceptualization, J.C. and Y.J.; Data curation, J.C.; Formal analysis, J.C., G.W. and Y.J.; Investigation, Y.J.; Methodology, J.C., G.W. and Y.J.; Project administration, Y.J.; Software, J.C.; Supervision, Y.J.; Validation, J.C.; Writing-original draft, J.C. and Y.J.; Writing-review \& editing, J.C., G.W. and Y.J. All authors have read and agreed to the published version of the manuscript.

Funding: This research received no external funding.

Institutional Review Board Statement: Not applicable.

Informed Consent Statement: Not applicable.

Data Availability Statement: Anyone who needs the data about this paper, please contacts us through cjhsy111@shu.edu.cn.

Conflicts of Interest: The authors declare no conflict of interest.

\section{References}

1. Bracken, J.; McGill, J.T. Mathematical programs with optimization problems in the constraints. Oper. Res. 1973, 21, 37-44. [CrossRef]

2. Emam, O.E. A fuzzy approach for bi-level integer non-linear programming problem. Appl. Math. Comput. 2006, 172, 62-71. [CrossRef]

3. Nicholls, M.G. The application of non-linear bi-level programming to the aluminium industry. J. Glob. Optim. 1996, 8, 245-261. [CrossRef]

4. Maher, M.J.; Zhang, X.; Vliet, D.V. A bi-level programming approach for trip matrix estimation and traffic control problems with stochastic user equilibrium link flows. Transp. Res. Part B Methodol. 2001, 35, 23-40. [CrossRef]

5. Yang, W.; Li, L.; Ma, S. Coordinating supply chain response-time: A bi-level programming approach. Int. J. Adv. Manuf. Technol. 2007, 31, 1034-1043. [CrossRef]

6. Jenabi, M.; Ghomi, S.M.T.F.; Smeers, Y. Bi-level game approaches for coordination of generation and transmission expansion planning within a market environment. IEEE Trans. Power Syst. 2013, 28, 2639-2650. [CrossRef]

7. Sadigh, A.N.; Mozafari, M.; Karimi, B. Manufacturer cretailer supply chain coordination: A bi-level programming approach. Adv. Eng. Softw. 2012, 45, 144-152. [CrossRef]

8. Amirtaheri, O.; Zandieh, M.; Dorri, B.; Motameni, A.R. A bi-level programming approach for production-distribution supply chain problem. Comput. Ind. Eng. 2017, 110, 527-537. [CrossRef]

9. Lv, Y.; Huang, G.H.; Li, Y.P.; Yang, Z.F.; Liu, Y.; Cheng, G.H. Planning regional water resources system using an interval fuzzy bi-level programming method. J. Environ. Inform. 2015, 16, 43-56. [CrossRef]

10. Keshavarz, E.; Khorram, E. A fuzzy bi-criteria transportation problem. Comput. Ind. Eng. 2011, 61, 947-957. [CrossRef]

11. Zhou, X.; Tu, Y.; Lev, B. Production and work force assignment problem: A bi-level programming approach. Int. J. Manag. Sci. Eng. Manag. 2015, 10, 50-61. [CrossRef]

12. Zhou, X.; Yu, N.; Tu, Y.; Pedrycz, W.; Lev, B. Bi-level plant selection and production allocation model under type-2 fuzzy demand. Expert Syst. Appl. 2017, 86, 87-98. [CrossRef]

13. Zhou, J.; Liu, B. Analysis and algorithms of bifuzzy systems. Int. J. Uncertain. Fuzziness Knowl.-Based Syst. 2004, 12, 357-376. [CrossRef] 
14. Liu, B. Toward fuzzy optimization without mathematical ambiguity. Fuzzy Optim. Decis. Mak. 2002, 1, 43-63. [CrossRef]

15. Zhou, J.; Liu, B. Bifuzzy programming and hybrid intelligent algorithms. In Proceedings of the Second International Conference on Information and Management Sciences, Las Vegas, NV, USA, 23-26 June 2003; Volume 2, pp. 440-445.

16. Wang, G.; Shen, Y.; Jiang, Y.; Chen, J. The scalar mean chance and expected value of regular bifuzzy variables. Symmetry 2021, 13, 1428. [CrossRef]

17. Dubois, D.; Prade, H. Fuzzy Sets and Systems: Theory of Applications; Academic: New York, NY, USA, 1980.

18. Liu, B. Theory and Practice of Uncertain Programming; Springer: Berlin, Germany, 2002.

19. Liu, B.; Liu, Y. Expected value of fuzzy variable and fuzzy expected value models. IEEE Trans. Fuzzy Syst. 2002, 10, 445-450.

20. Zhou, J.; Yang, F.; Wang, K. Fuzzy arithmetic on LR fuzzy numbers with applications to fuzzy programming. J. Intell. Fuzzy Syst. 2016, 30, 71-87. [CrossRef]

21. Liu, B. Uncertainty Theory: A Branch of Mathematics for Modeling Human Uncertainty; Springer: Berlin, Germany, 2010.

22. Bard, J. Practical Bilevel Optimization: Algorithms and Applications; Kluwer Academic Publishers: Dordrecht, The Netherlands, 1998.

23. Roghanian, E.; Aryanezhad, M.B.; Sadjadi, S.J. Integrating goal programming, Kuhn-Tucker conditions, and penalty function approaches to solve linear bi-level programming problems. Appl. Math. Comput. 2008, 195, 585-590. [CrossRef]

24. Han, J.; Zhang, G.; Hu, Y.; Jie, L. A solution to bi/tri-level programming problems using particle swarm optimization. Inf. Sci. 2016, 370, 519-537. [CrossRef]

25. Sun, H.; Gao, Z.; Wu, J. A bi-level programming model and solution algorithm for the location of logistics distribution centers. Appl. Math. Model. 2008, 32, 610-616. [CrossRef]

26. Ghomi-Avili, M.; Tavakkoli-Moghaddam, R.; Naeini, S.G.J.; Jabbarzadeh, A. Competitive green supply chain network design model considering inventory decisions under uncertainty: A real case of a filter company. Int. J. Prod. Res. 2021, 59, 4248-4267. [CrossRef] 